Vol. 26, No. 01, pp. 34-44/Junio 2013

\title{
Evaluación de riesgos en sistemas agrícolas asociados a la utilización de plaguicidas en el Municipio de Kukra Hill, Nicaragua, Centroamérica
}

\author{
A. Flores-Pacheco ${ }^{* 1}$, A. Mairena ${ }^{1}$, J. Espluga $^{2}$ \\ ${ }^{1}$ Facultad de Recursos Naturales y Medio Ambiente, Bluefields Indian \& Caribbean University- BICU \\ Apartado postal $\mathrm{N}^{\circ} 88$, Avenida Universitaria, Bluefields, Nicaragua \\ ${ }^{2}$ Departamento de Sociología, Universidad Autónoma de Barcelona, España \\ Campus de la UAB; 08193 Bellaterra \\ E mail asdrubalflorespacheco@gmail.com, amava7@gmail.com, joseplluis.espluga@uab.cat
}

(Recibido/received: 24-Mayo-2013; aceptado/accepted: 30-Junio-2013)

\begin{abstract}
RESUMEN
La presente investigación pretende identificar y tipificar los riesgos humanos y ambientales asociados a la utilización de plaguicidas en las actividades agrícolas en el municipio de Kukra Hill, Región Autónoma del Atlántico Sur (RAAS), Nicaragua. Se trabajó desde la evaluación de sus concentraciones en suelos y escorrentías superficiales en áreas de uso agrícola, lo que es complementado con una investigación de las condiciones de seguridad laboral, tanto preventiva como correctiva, que los agro-aplicadores realizan en sus labores diarias. Se logró determinar que existen presencia y concentración de moléculas de plaguicida en concentraciones por debajo de los niveles de detección permisibles según valores guías canadienses de calidad de suelo de uso agrícola para la protección ambiental y salud humana. Se han incluido los testimonios de técnicos extensionistas privados y de instituciones gubernamentales. Los resultados muestran un escaso uso de protecciones personales y una importante exposición laboral y extra-laboral a pesticidas.
\end{abstract}

Palabras claves: Plaguicidas; riesgos humanos y ambientales; seguridad laboral; análisis en suelos y aguas.

\begin{abstract}
The present reach's is intended to identify and to categorize the human risks and acclimatize associates to the utilization of pesticides in the agricultural activities at Kukra Hill's municipality, South Atlantic Autonomous Region (RAAS), Nicaragua. He was worked up from the evaluation of his concentrations at grounds and superficial run-offs in areas of agricultural use, what is complemented with a labor investigation of safeness's, so much yellow light like corrective, than them agriculture the applicators accomplish in their daily works. Presence was possible to be determined that there exists and concentration of molecules of pesticide in concentrations underneath the levels of detection permissible according to values you drive Canadian of quality of ground of agricultural use for the environmental protection and human health the private and governmental- institutions agronomists Have Included technicians' testimonies themselves. Results evidence a scarce use of personal protections and an important labor and extra-labor exposure to pesticides.
\end{abstract}

Key words: Pesticides; human and environmental risks; job safety; analysis in soils and waters.

\footnotetext{
* Autor para la correspondencia
} 


\section{A. Flores-Pacheco, A. Mairena, J. Espluga}

\section{INTRODUCCIÓN}

En Nicaragua, como otros países agroexportadores en Centroamérica, el consumo de agroquímicos ha aumentado en las últimas décadas, con el afán de mejorar la tecnología agrícola e incrementar la producción (Miller, 2004).

Hoy en día, se puede calificar de masivo el uso y abuso de estos productos por parte de los agricultores, quienes han llegado a depender casi totalmente de estos compuestos. Este empleo intensivo está produciendo efectos negativos a nivel económico, agrícola, ambiental y en la salud (Hilje et al, 1987). Más de 30,000 toneladas de residuos tóxicos de agroquímicos se usan en América Latina, y la magnitud de uso de los residuos químicos tóxicos provenientes de plaguicidas obsoletos en Latinoamérica es mucho más amplia de lo que se había estimado previamente (FAO, 2007).

Las consecuencias económicas negativas, pueden ser divididas en costos directos e indirectos, y están estrechamente relacionadas con el efecto de los agroquímicos en la salud, el ambiente y la agricultura y por ende en la seguridad alimenticia de los pueblos (Bull, 2006). Las consecuencias ambientales son también cada vez más evidentes. La agricultura moderna se enfrenta a una crisis ambiental cuyos orígenes radican, en parte, en las prácticas agrícolas intensivas (inadecuadas) basadas en altos insumos agroquímicos, que conllevan la degradación de los recursos naturales a través del sobre-laboreo del suelo, el uso irracional de plaguicidas, o las prácticas repetidas de quema de rastrojos, que se traducen en continuas reducciones de la productividad (Wesseling, 1994).

Las consecuencias sobre la salud están relacionadas con el uso y exposición de las personas. La exposición prolongada y periódica a plaguicidas puede causar intoxicaciones agudas o crónicas, dependiendo del tiempo de la misma y de la cantidad a la que la persona se expone. Las intoxicaciones crónicas resultan de la entrada al organismo de bajas cantidades de plaguicidas, pero en forma repetida y prolongada siendo el mayor peligro por el tipo de trabajo realizado conjugado con el carente uso de equipos de protección personal (Mamani y Merjildo, 2002).

Las lecciones aprendidas de estas experiencias, permiten afirmar que las tecnologías agrarias deberían aplicarse considerando las condiciones apropiadas para el establecimiento y buen desarrollo de las especies recomendadas en un contexto agroecológico determinado; se debe considerar además de estas variables, la tipología de los agricultores a quienes se les recomienda determinada tecnología (Hilje, 1987).

El objetivo de la investigación ha sido estimar la exposición a plaguicidas de una muestra de trabajadores agrícolas, mediante el análisis de la presencia de plaguicidas en el entorno en el que trabajan y viven, así como mediante el análisis de sus percepciones y prácticas de riesgo y de uso de medidas protectoras y preventivas. La finalidad última es formular recomendaciones para un uso más sostenible y seguro de los agroquímicos entre dicha población.

\section{METODOLOGÍA}

\section{Ubicación del proyecto}

La ubicación del proyecto fueron las comunidades del Municipio de Kukra Hill principalmente los territorios de las comunidades Big Lagoon, Samuel Law, El Panchón, y La Fonseca, RAAS, Nicaragua.

\section{Generalidades del Municipio de Kukra Hill.}

La zona de estudio se caracteriza por presentar un clima tropical húmedo de selva con temperaturas que oscilan entre $24{ }^{\circ} \mathrm{C}$ y $30^{\circ} \mathrm{C}$. Se le considera una zona húmeda basada en la clasificación de zonas de vida de Holdridge, con precipitaciones anuales de 2,000 a 4,000 mm distribuidas de 9 a 10 meses, siendo el mes más lluvioso el de mayo. La región es baja y pantanosa, a lo largo de la costa no excediendo los $30 \mathrm{msnm}$. Se ubica entre las coordenadas 12"14" Latitud Norte y 83"45' de Longitud Oeste (Alcaldía Municipal de Kukra Hill, 2007).

\section{Técnicas utilizadas.}

Se han utilizado varias técnicas de investigación, tanto para el análisis de las percepciones y comportamientos de la población (encuestas por cuestionario, entrevistas en profundidad, análisis documental y observación no participante), como para el análisis de residuos de plaguicidas en aguas y suelos, tal como a continuación se describen. 
Monitoreo de plaguicidas en suelos y aguas de escorrentías superficiales. ${ }^{4}$

Se estimó la concentración de moléculas de plaguicida en suelo y en aguas de escorrentías superficiales en parcelas del cultivo de palma africana. Pudiendo identificar los tipos de plaguicidas, sus niveles de concentración y asociarlo a los posibles riesgos ambientales y humanos que estos conllevan en combinación con las condiciones de seguridad laboral.

\section{Colecta de muestra de suelo}

Las muestras de suelo fueron colectadas llenando frascos de vidrio con $1 \mathrm{Kg}$ de suelo, utilizando espátulas de aluminio. Se colectó una muestra compuesta de 200 $\mathrm{mg}$ por cinco 5 sub-muestras a una profundidad de 0.2 metros. Posteriormente se limpió el borde del frasco con un pañuelo desechable, se ubicó papel de aluminio sobre su boca y se colocó su respectiva tapa con cinta adhesiva eléctrica. Estos procedimientos (PROC-CO-05) establecidos por el laboratorio de contaminante orgánicos del CIRA/UNAN.

\section{Colecta de muestras de aguas de escorrentías}

Se procedió a recolectar las aguas de escorrentías a nivel superficial, se utilizó beaker para colectar las muestras de agua de forma diagonal depositándose en botellas de vidrios esterilizados y envueltos en papel aluminio. Se rotuló cada frasco con la muestra, se depositaron en un termo con hielo para garantizar la calidad de las muestras. Estas fueron enviadas al laboratorio del Centro de Investigación de Recursos Acuáticos de la Universidad Nacional Autónoma de Nicaragua (CIRAUNAN) para realizar sus respectivos análisis. El volumen de cada muestra para el análisis de plaguicidas fue de 2 litros $(2,000 \mathrm{ml})$.

\section{Cálculo de la dosis suministrada}

La magnitud de la exposición se determina estimando la cantidad (concentración) del agente que está presente en la superficie de contacto (pulmones, intestino, piel, etc.) durante un período especificado. Esta cantidad cuando se expresa por unidad de masa corporal del individuo expuesto (agro-aplicador). Para ello se analizan factores tales:

\footnotetext{
${ }^{4}$ En base Valores guía canadiense de calidad de suelos de uso agrícolas para la protección ambiental y la salud humana (CCME), 2002
}

\author{
Ds $=($ CTFD $) /($ MP $)\left[\right.$ Ecuación $\left.\mathrm{N}^{\circ} 1\right]$ \\ Dónde: \\ $\mathbf{C}=$ Concentración promedio durante el período de \\ exposición \\ $\mathbf{T}=$ Tasa de contacto, la cantidad de medio contactado \\ por unidad de tiempo \\ $\mathbf{F}=$ Frecuencia de exposición \\ D = Duración \\ $\mathbf{M}=$ Masa corporal \\ $\mathbf{P}=$ Tiempo de promediación
}

\section{Técnicas de investigación social:}

Se han utilizado encuestas y entrevistas, además de análisis documental y observaciones de campo.

a) Encuestas a población agrícola.

$\mathrm{Se}$ han realizado dos encuestas mediante sendos cuestionarios:

- Encuesta dirigida a productores agrícolas independientes de palma africana en el municipio de Kukra Hill (Encuesta tipo I).

- Encuesta condiciones de manejo de plaguicidas por parte de los aplicadores de cultivos de palma africana, Kukra Hill (Encuesta tipo II).

\section{Muestra:}

Se tomó una muestra de 220 individuos, correspondiente al $44 \%$ de la población total de la zona (con un error de $\pm 5 \%$ para un nivel de confianza del $95,5 \%$ ). Las comunidades a seleccionar para la investigación, donde residen de manera permanente productores independientes los cuales utilizan plaguicidas en sus actividades agropecuarias (BICU, 2010).

\section{Criterios de inclusión:}

Presentar disponibilidad y voluntariedad del suministro de información. Poseer conocimiento de las características y condiciones de la comunidad y/o Agroecosistemas. Vivir en algunas de las comunidades dentro de la zona de incidencia de la investigación. Ser productor o técnico extensionista del municipio de Kukra Hill. Utilizar agroquímicos en las labores agrícolas de que ejecuta en sus cultivos.

Procedimiento de análisis:

La sistematización de la información se efectuó en una base de datos en SPSS 20.0. Al sistema se solicitó la distribución por frecuencia (porcentajes) y comportamiento de los datos que se recolectaron (media, 
varianza y desviación estándar). Estos resultados datos se expresan en resúmenes de casos en tablas y gráficos.

b) Entrevistas a expertos.

Por otro lado, se ha llevado a cabo una serie de 21 entrevistas en profundidad a técnicos agrónomos que ejercen funciones de asesoramiento a los productores agrícolas en materia de lucha contra las plagas, y personas clave que conocen aquella realidad social a fin de conocer su dominio del tema en cuestión, sus experiencias con los productores y la opinión que estos poseen sobre los riesgo del uso de plaguicidas. Se trata de técnicos privados y gubernamentales expertos en la temática, de las siguientes instituciones: Instituto Nicaragüense de Tecnología Agropecuaria (6), Ministerio Agropecuario y Forestal (10), Ministerio de Recursos Naturales (1), Universidades (3), Alcaldía de Kukra Hill (1).

c) Otras técnicas de investigación social.

Finalmente, se ha realizado un análisis de documentos generados por las empresas y municipalidades del lugar, así como observaciones sistemáticas durante el trabajo de campo.

\section{RESULTADOS Y DISCUSIÓN}

En primer lugar se indican los resultados del análisis del entorno en el que los trabajadores viven y trabajan (aguas y suelos), mientras que en segundo lugar se describen los resultados referidos a sus percepciones de riesgo y comportamientos de seguridad.

\section{Resultados del análisis de aguas y suelos}

Análisis de riesgos por la presencia y concentración de plaguicidas en muestras de aguas en escorrentías superficiales.

Como resultado del análisis de las muestras de aguas tomadas en las comunidades de Kukra Hill, se detectaron algunos plaguicidas organoclorados, organofosforados y carbamatos como se expresa en la tabla $\mathrm{N}^{\mathrm{o}} 1$.

Tabla $\mathbf{N}^{\mathbf{0}}$ 1: Plaguicidas detectados en muestras de escorrentía de las parcelas del cultivo de la palma africana en el municipio de Kukra Hill $\left(\mathrm{ng} / \mathrm{g}^{-1}\right)$.

\begin{tabular}{|l|c|c|c|c|c|}
\hline \multicolumn{1}{|c|}{ Plaguicidas detectados } & $\begin{array}{c}\text { Límite de } \\
\text { detección } \\
\text { ng/g-1 }\end{array}$ & Big Lagoon & $\begin{array}{c}\text { Flor de } \\
\text { Pino }\end{array}$ & Samuel Law & La Fonseca \\
\hline Organoclorados & 0.09 & 1,27 & $\mathrm{Nd}$ & $\mathrm{Nd}$ & $\mathrm{Nd}$ \\
\hline Alfa-HCH & 0.08 & $\mathrm{Nd}$ & 4,59 & $\mathrm{Nd}$ & $\mathrm{Nd}$ \\
\hline Beta-HCH & 0.09 & 3,27 & $\mathrm{Nd}$ & 20,19 & $\mathrm{Nd}$ \\
\hline Aldrin & 0.16 & $\mathrm{Nd}$ & 5,14 & $\mathrm{Nd}$ & $\mathrm{Nd}$ \\
\hline Endosulfán sulfato & \multicolumn{7}{|c|}{} \\
\hline Organofosforado & 0.3 & $\mathrm{Nd}$ & $\mathrm{Nd}$ & 8,49 & $\mathrm{Nd}$ \\
\hline Etil Paratión & \multicolumn{7}{|l|}{} \\
\hline Carbamatos & 41.3 & $\mathrm{Nd}$ & $\mathrm{Nd}$ & $\mathrm{Nd}$ & 66,61 \\
\hline Aldicarb & 4.28 & $\mathrm{Nd}$ & $\mathrm{Nd}$ & 57.03 & 28,25 \\
\hline Oxamil
\end{tabular}

Fuente: Elaboración propia. [Nd: No detectado]

Se infiere que la presencia de estos plaguicidas se debe a su uso intensivo en la industria y agricultura del municipio, ya que desde la década de los sesenta ha sido un enclave agrícola con el establecimiento de un emporio bananero ( $\mathrm{Li}$ et. al., 2001), posteriormente en la década de los ochenta se establece un ingenio azucarero $\mathrm{y}$, a partir de la década de los noventa y hasta la fecha actual, se da un cultivo intensivo de palma africana. En todos estos cultivos se han utilizado una serie de productos que hoy en día se categorizan como contaminantes orgánicos persistentes (COP's) tales como son Hexaclorociclohexanos (HCH's) y diclorodifenil-tricloroetano (DDT), así como otros plaguicidas organoclorados, utilizados principalmente para controlar plagas agrícolas y enfermedades transmitidas por insectos vectores (Doong et al., 2002; Kishimba et al., 
2004). La utilización de estos compuestos puede provocar la contaminación del agua, sedimentos así como organismos acuáticos y terrestres de no dárseles el manejo adecuado representando un elevado peligro ambiental y humano. El conocimiento de las concentraciones de estos contaminantes proporciona información básica para que los gobiernos puedan tomar las medidas pertinentes para su control (Ioannis et al., 2006) Ante la ausencia de suelos ricos en materia orgánica (Flores y Zavala, 2012) es de suponerse que los metabolitos del DDT (4,4'-DDE) producto de las escorrentías son arrastrados al mar $\mathrm{y}$, por ser bioacumulables, dado que su persistencia media en el agua es mayor de un año (Sarkar et al., 2008;)., se constituyen en un riesgo para la fauna acuática y para la salud de personas ante el consumo de productos procedentes de estos ecosistemas, ya que todos los organismos pierden agua al ambiente, los contaminantes se pierden junto con el agua, pero la grasa no se pierde (Malik et al., 2010).
El Endosulfán y Endosulfán Sulfato también por su persistencia y dificultad de infiltración subterránea, ya que los suelos de la zona son desde mediana hasta altamente compactos por el régimen lluvioso de la región, que oscila entre los 2,000 y 2,500 mm según INETER en 2002, dichas precipitaciones y la erosión de los suelos, especialmente en las áreas con parcelas ubicadas en altas pendientes en Kukra Hill, dan origen a arrastre de sedimentos (partículas de suelo y materia orgánica conteniendo moléculas y metabolitos de plaguicidas) hacia el mar y otros cuerpos de aguas superficiales (Carvalho et al, 2002).

Análisis de riesgos por la presencia y concentración de plaguicidas en muestras de suelos.

Tabla $\mathbf{N}^{\circ}$ 2: Resumen de resultados de análisis de plaguicidas en muestras de suelo expresados en $\mathrm{ng} / \mathrm{g}^{-1}$.

\begin{tabular}{|l|c|l|l|l|l|l|}
\hline $\begin{array}{c}\text { Plaguicida } \\
\text { detectados }\end{array}$ & $\begin{array}{c}\text { Límite de } \\
\text { detección } \\
\text { ng/g }\end{array}$ & $\begin{array}{c}\text { Big } \\
\text { Lagoon }\end{array}$ & $\begin{array}{c}\text { Flor de } \\
\text { Pino }\end{array}$ & $\begin{array}{c}\text { Samuel } \\
\text { Law }\end{array}$ & $\begin{array}{c}\text { La } \\
\text { Fonseca }\end{array}$ & $\begin{array}{c}\text { Valores guías } \\
\text { ng/g-1 }\end{array}$ \\
\hline Insecticidas organoclorados \\
\hline $4,4^{\prime}$-DDE & 0.06 & 0,53 & nd & 0,42 & nd & 700.00 \\
\hline $4,4^{\prime}$-DDD & 0.03 & nd & nd & nd & nd & 700.00 \\
\hline $4,4^{\prime}$-DDT & 0.06 & nd & nd & nd & nd & 700.00 \\
\hline DDT total & 0.06 & nd & nd & nd & nd & 700.00 \\
\hline Endosulfán & 0.1 & nd & 0,57 & nd & nd & 700.00 \\
\hline $\begin{array}{l}\text { Endosulfán } \\
\text { sulfato }\end{array}$ & 0.16 & nd & 2,06 & nd & nd & 700.00 \\
\hline
\end{tabular}

Fuente: Elaboración propia. [Nd: No detectado]

En las muestras de suelo dedicadas al cultivo de la palma africana en ninguno de los cuatro sitios muestreados en las comunidades Big Lagoon y La Fonseca, no se detectó presencia reciente de moléculas perteneciente a los grupos de los insecticidas organofosforados y agroquímicos organofosforados

En las muestras de suelo dedicadas al cultivo de la palma africana en ninguno de los cuatro sitios muestreados en las comunidades Big Lagoon y La Fonseca, no se detectó presencia reciente de moléculas perteneciente a los grupos de los insecticidas organofosforados y agroquímicos organofosforados.
La ausencia de residuos de plaguicidas obedece a factores físico-climatológicos, entre otros. La ausencia de estos grupos de plaguicidas en las parcelas de las

Comunidades Big Lagoon y La Fonseca, según CIRAUNAN, "indica que estos compuestos han sido poco utilizados en el cultivo de la palma africana, la cantidad aplicada fue tal que las concentraciones en el suelo son extremadamente bajas y no son instrumentalmente detectables, se han degradado con el tiempo o posiblemente se han lixiviado o escurrido incorporándose en el agua subterránea o superficial respectivamente a causa de las pendientes de las parcelas" (BICU, 2001; Pp. 12). Las altas precipitaciones (más de 2,500 mm/año) que caen en la zona podrían estar facilitando la migración casi 
instantánea de los plaguicidas posteriormente a su aplicación. De ahí la no detección en los muestreos de suelo, los cuales fueron tomados en época seca. Este análisis podría fortalecerse si se aplicases muestreos en época lluviosa, lo cual permitiría valorar el escurrimiento de plaguicidas y el análisis de riesgo en una mayor dimensión. La gran afinidad de los contaminantes por el material particularmente fino, como es el caso de los suelos arcillosos de Kukra Hill, permite que estos puedan ser registrados en la columna de sedimento, ya que los COP's tienen una limitada solubilidad en agua y un elevado coeficiente de partición hacia el material en suspensión, existiendo una estrecha relación entre los contaminantes y los niveles de materia orgánica presentes en los sedimentos. Los sedimentos pueden actuar como portadores y posibles fuentes de contaminación porque los contaminantes no quedan permanentemente retenidos y pueden ser liberados a la columna de agua por cambios en las condiciones ambientales tales como $\mathrm{pH}$, potencial redox, oxígeno disuelto o la presencia de coloides orgánicos Sigg et al., 1987, Singh et al., 1999, citado por Romero (2010).

PNUMA en 2011 manifiesta que un eventual escurrimiento de plaguicidas con la adición de sales en el medio marino y cuerpos dulceacuícolas como el Rio Kama, Malopy, El Panchón, etc., que desembocan en el río escondido, transformaría el ecosistema acuático en perjuicio de la fauna acuática, también la reproducción y fisiología de los manglares, por cuanto un aumento en la salinidad alteraría el sistema estuarino especialmente en los meses de menor precipitación y, por ende, la cadena alimenticia vinculada al ecosistema manglar. Los plaguicidas identificados y que quedan en los suelos pueden afectar las propiedades químicas de este y el equilibrio de sus poblaciones. La degradación en el suelo puede ser biológica, química o fotoquímica. Sin embargo, la degradación puede presentar diversos problemas como: resistencia a la degradación biológica, así ocurre con los organoclorados que mantiene su actividad por largo tiempo, o puede ser incompleta y sus metabolitos persistir en el ambiente. También pueden existir factores ambientales o interacciones que obstaculicen la descomposición de tóxicos biodegradables (Albert, 2001).

\section{Resultados del análisis de las percepciones de la población y expertos}

Características de la población objeto de estudio:

Los 220 encuestados, tanto de sexo masculino (90.6\%) como femenino $(9.4 \%)$, con edades comprendidas entre
18-67 años (edad media 35 años, 4,7\% menores de 21 años y $6 \%$ mayores de 55 años), con un estado civil predominante de unión libre. Las edades comprenden la etapa productiva, siendo estos cabezas de familia, lo que indica alto grado de responsabilidad. La historia Laboral de los agricultores que conforman la muestra analizada comprende periodos mínimos de trabajo 4 años y máximo de 40 años con una media de 10 años. Los trabajadores dedicados a la agricultura suelen estar sistemáticamente menos protegidos que los que participan laboralmente del resto de las actividades económicas, integrando en consecuencia, las poblaciones más vulnerables frente a los riesgos del trabajo; son los asalariados más desfavorecidos y presentan serias dificultades en su forma de vida, cultura y educación, carecen de organizaciones laborales, sindicales, por ende poca asesoría y falta de conocimiento sobre la legislación laboral de acuerdo a lo manifestado en las entrevistas a productores, agroaplicadores y técnicos coincidiendo con Galli (2002).

Exposición individual y familiar:

Se detecta que, a pesar de ser una actividad familiar, sólo los hombres se dedican a la fumigación y a la recolección de las cosechas, mientras las mujeres se dedican al cuido de la casa y los niños. Se cree que estas tendencias pueden deberse al transporte de los equipos, los cuales son pesados. No obstante, la remuneración según el rendimiento llamado en algunos países "trabajo a destajo" o "por tanto", es una modalidad frecuente, en especial en tareas mencionadas de poda, tala, fumigación, recolección o cosecha. Cuando se trabaja bajo este sistema, es frecuente que el trabajador recurra a la colaboración del grupo familiar, impactando ello en la realidad del trabajo de las mujeres incorporado a la agricultura y el de los niños o jóvenes (Mamani y Merjildo, 2002).

\section{Percepción de riesgos}

Durante el trabajo de campo, los agricultores encuestados permitieron recabar información que los trabajadores tenían sobre los accidentes laborales y enfermedades profesionales bajo cuyos riesgos se encontraban, indagando mediante preguntas abiertas. Los mencionados informan que si bien hay variaciones a nivel de cada sub-sector analizado (trabajador agrícola, contratista agrícola, productor y extensionista), el grueso de las respuestas se centró en los accidentes que se producen por el manejo inadecuado de agroquímicos y el empleo de herramientas y/o maquinarias utilizadas cotidianamente en las labores agropecuarias. Se reviste 
de importancia la percepción que tienen los trabajadores respecto de la incidencia de los accidentes originados por el manipuleo de agroquímicos. Quizás en las respuestas recabadas haya un solapamiento entre las referencias a los accidentes producidos por este manejo y los generados por el empleo de ciertas maquinarias e implementos, pues buena parte de las referencias a estos últimos tienen que ver solo con los aplicadores de insecticidas y herbicidas. Un aspecto importante que surge es la escasa percepción de los trabajadores acerca de la presencia de enfermedades profesionales en la actividad agropecuaria (100\%). La mayoría desconocen que los procedimientos mal aplicados en sus labores cotidianas al combinarse con otros factores son los agentes potenciales de enfermedades y problemas de salud. En las entrevistas, los trabajadores mencionan reiteradas veces que sí conocen que años atrás había problemas con ciertas enfermedades, en especial mencionan a la "flecha seca", que actualmente está erradicada en muchas plantaciones de palma africana del municipio. Más allá de esta referencia, y de unas pocas a ciertas zoonosis relativas a la ganadería, no aparecen expresiones que pongan el acento en la cuestión de la enfermedad profesional, sino que toda la atención está centrada en los accidentes laborales. Esto coincide con lo encontrado en la bibliografía especializada (Wrigth, 2002). La información que brindaron, podría extrapolarse al resto de las realidades de la región, pues en la mayoría de los casos la percepción del riesgo que tienen los trabajadores en el agro es la de que éste es algo natural y al que aceptan en muchos casos como algo inevitable. Asimismo, hay una actitud frecuente de "Nunca me sucedió nada y siempre lo hice así", suele ser el tipo de frases que acuñan ante la advertencia de una situación de riesgo en una tarea, en la que es necesario adoptar una modalidad segura.

Tipificación de las condiciones laborales del uso de plaguicidas en el municipio de Kukra Hill.

De acuerdo con los datos recopilados en el presente trabajo, la exposición de los trabajadores a los plaguicidas es como sigue:

Condiciones y dosis de exposición a plaguicidas:

- El 56,3\% de los trabajadores tienen una antigüedad en su tarea mayor a los 5 años.

- Más de la mitad de los sujetos trabajó una superficie anual aproximada de hasta $500 \mathrm{Ha}$ en el último año.

- El equipo de aplicación utilizado en un $100 \%$ es la bomba de mochila o espalda. El tiempo de exposición promedio es de 8 horas diarias.
Aquellos que laboran como fumigadores en la Cukra Development Corporation S.A. (CDC) realizan esta labor durante 5 días por semana, para los trabajadores independientes dependerá de las actividades y extensión de la finca en la cual laboran.

- Dosis suministrada (mg/Kg/día de aplicación) obtenida en base a las cantidades de pesticidas aplicados por unidad de área y tiempo empleado por cada trabajador agrícola y la relación por unidad de masa y tiempo de aplicación en un área promedio de una hectárea: Se calculó en $2.195 \mathrm{mg} / \mathrm{Kg} /$ día por aplicación. Este hecho combinado con el nulo o inadecuado uso de equipos de protección personal y el indistinto manejo de dichas ropas al combinarse con el resto de ropas de la familia aumenta significativamente el riesgo de intoxicaciones y bioacumulación por acción de los plaguicidas tanto de forma directa como cruzada.

- Las sustancias químicas más utilizadas son el glifosato (roundup), productos de la familia del bipiridilo (Gramaxone) y clorpirifos (Lorban). Por su nombre genérico fueron Gramaxone (41\%), Glifosato 2-4-D y Cypermetrina, todos con un $17 \%$ de uso, entre muchos otros. Se procedió a clasificarlos según el grado de restricción de su registro dado por el OMS/OPS y se observó que el $44.9 \%$ eran de uso restringido y el $5.1 \%$ extremadamente peligros clasificándose en la categoría I-A.

Las vías de exposición al contaminante, que sirven en el análisis de la ruta de exposición, corresponden prioritariamente a las vía dérmica o cutáneo-mucosa y la vía inhalatoria (Turnbull et al., 1985); la primera de éstas es la más importante en relación a la cantidad de producto absorbido (Vílchez., 2009). Así, la utilización de ropa impermeable es una de las medidas prioritarias a tomar en prevención, ya que la penetración a través de las prendas de protección específica es mínima. Según los datos analizados:

- La combinación de elementos de protección mínimos necesarios para estar protegidos, esto es, ropa impermeable, careta antigás y guantes químicamente resistentes, sólo fue implementada de manera rutinaria por $11,6 \%$ de los trabajadores y $29 \%$ de ellos se mostraron sólo parcialmente protegidos. Nótese que el resto, que alcanzó 59,3\%, no se encontró protegido en absoluto. 
- El tipo de exposición predominante es por inhalación y contacto, puesto que en el $90 \%$ de los casos solo utilizan botas y vestido normal $(100 \%)$. Esto indica la no utilización de los elementos de protección personal, ni las indicaciones adecuadas para el desempeño de esta labor (Galli, 2002 y WorkingLife, 1999).

Por lo tanto, se observa que ninguno (100\%) de los trabajadores se viste con ropa impermeable para sus faenas y tampoco utilizan caretas antigás ni protectores de cara; y que la proporción de usuarios de guantes químicamente resistentes es sólo ligeramente superior. La combinación de los elementos de protección mínimos necesarios para ser considerados "protegidos", esto es, ropa impermeable, careta antigás y guantes químicamente resistentes, sólo es implementada de manera rutinaria por el $40 \%$ de los trabajadores, mientras que el resto, que alcanza al $60 \%$, no se encuentra protegido, acorde a la valoración de protección personal desarrollada en este estudio.

Es de resaltar que los datos recolectados por medio de la aplicación de encuestas a los agro-aplicadores mostraron variación en cuanto al grado de protección destacando estas:

- $\quad$ Nivel de instrucción: se encontraron protegidos en menor proporción los trabajadores con menor nivel de instrucción $(\mathrm{p}<0,05)$.

- Estado civil: se protegieron con más frecuencia aquellos casados o unidos de hecho $(\mathrm{p}<0,05)$. En el caso de los trabajadores que están en pareja, la visión a la autoprotección puede deberse a una mayor responsabilidad familiar, o a una participación directa de la familia en la decisión de la utilización de equipo de protección personal, que los conduce a cuidarse más eficientemente frente a los riesgos ocupacionales. Si esto fuera real, se tendría un aspecto importante a tener en cuenta en la planificación de las estrategias de prevención.

- Superficie cultivada: La protección adecuada se ha asociado negativamente a la cantidad de hectáreas trabajadas $(\mathrm{p}<0,01)$

- Tipo de equipo de aplicación ( $\mathrm{p}<0,01)$ mochila o bomba de espalda (100\% de uso en todos los casos).

- Existencia de asesoramiento experto: Se asoció también a la modalidad en la indicación de uso de plaguicidas por parte de un profesional ingeniero agrónomo, en una receta indicada $(\mathrm{p}<0,05)$.
- Período del año y las variaciones de temperatura: en los meses de mayor temperatura el valor de RR (riesgo relativo) de estar protegido total o parcialmente respecto de no estar protegido fue igual a 0,71 , y de 0,80 en los meses de menores temperaturas.

En el contexto descrito, la utilización de maquinarias con filtros y empaques de seguridad se asocia a un mayor uso de equipo de protección personal (EPP). Se podría decir que la provisión de este tipo de tecnología más segura muestra una preocupación por parte de los productores por el mejoramiento de las condiciones laborales del colectivo de trabajadores que tienen a cargo. No obstante, será necesario desarrollar futuros estudios para avanzar en el conocimiento del impacto de los entornos laborales y los modos de organización que los rodean, en la salud de la población de trabajadores. Algo similar ocurre con la aplicación de la receta fitosanitaria, como modalidad habitual de procedimiento de aplicación de productos agroquímicos. La intervención del ingeniero agrónomo a través de la prescripción de una receta fitosanitaria quizá refleje entornos laborales más controlados y seguros para el trabajador. Así, el rol del ingeniero agrónomo jugaría un papel clave en la protección de los trabajadores, probablemente, al promover la utilización de EPP.

\section{Exposiciones adicionales al momento de la aplicación:}

Además de la exposición directa a plaguicidas que los trabajadores sufren en el momento de la preparación y aplicación de los mismos, existen otras situaciones que prolongan dichas exposiciones y que pueden incrementar los riesgos considerablemente (no sólo para el trabajador, sino también para sus familiares):

a) Lugar de residencia:

Se observa que el $30 \%$ de los trabajadores vive a una distancia de 100 metros o menos de la zona de cultivo más próxima y el $49 \%$ de ellos están en un radio de 500 metros. Entre otras cosas, esto puede indicar que la exposición, una vez terminada la jornada laboral, tiene probabilidades de prolongarse. Además, es conocido que, en muchos casos, la familia del productor aporta mano de obra propia al sistema productivo, lo que trae aparejado una exposición ocupacional y paraocupacional de todo el grupo familiar. Esto incorpora grupos altamente vulnerables, como son la mujer en edad fértil y los niños, en todas sus etapas de crecimiento, los cuales deben tenerse en cuenta tanto en la evaluación de la problemática, como en las estrategias de abordaje. 
b) Manejo de envases vacíos de plaguicidas. En una entrevista realizada al Ingeniero Agroforestal que brinda sus servicios como extensionista de un ente gubernamental en la zona de Kukra Hill y Laguna de Perlas comenta:

"El triple lavado no sabe cómo debe hacerse ni qué significa. La mejor recomendación es destruirlo pero no se hace, entonces uno ve que los cortan por la mitad para darle agua o comida a los perros, a las gallinas. También se aconseja reciclarlos y uno ve que los envases se guardan hasta con etiqueta porque hay quienes que compran el envase y ponen por ejemplo glifosato (falso) en bidones donde la etiqueta no se rompió y lo venden a mitad de precio. Es muy difícil de controlar qué se hace con los envases porque es increíble la cantidad que llevan a los campos para hacer las aplicaciones." (Se mantiene la confidencialidad del entrevistado a fin de proteger su estatus laboral).

Formación y conocimiento de los riesgos y su prevención

Una desventaja identificada es el desconocimiento, la falta de capacitación y poco entendimiento de las instrucciones de los diferentes productos agrícolas por el bajo grado educativo. En este sentido, se observa que la mayoría de las personas encuestadas $(43.66 \%)$ tienen un nivel de educación de primaria incompleta, mientras que más de una cuarta parte (28.17\%) son analfabetas. Sólo la cuarta parte restante tiene educación primaria completa o más alta.

La bibliografía advierte que la capacitación del trabajador agrícola para el trabajo seguro suele encontrar dificultades para su implementación, por la dispersión de los establecimientos, el difícil acceso a los predios, porque los trabajadores no pueden abandonar las tareas y por la baja cantidad de personas en los campos. Estos dos últimos aspectos, posicionan el desarrollo de iniciativas de capacitación en prevención de riesgos con modalidades informales de acción en terreno o las técnicas de capacitación acción, de alta eficiencia pues permiten capacitar al trabajador en el mismo momento en el que realiza su trabajo (Rodríguez y Ramos, 1996), técnica que fue adoptada por el equipo de extensionista del proyecto REPCar-BICU durante el periodo 20102011, teniendo como mayor debilidad la corta duración de la fase de ejecución de campo (BICU, 2010) lo que generó expectativas en la población que al ver el rápido retiro del proyecto se diseccionó llevando en la mayor parte de los casos al caso omisos de las técnicas transferidas por dicho personal.
Formación de los técnicos asesores en materia de riesgos:

Otro aspecto a atender es la falta de adecuada formación de los técnicos del sector agropecuario, acerca de cómo atender la compleja problemática de la seguridad laboral en la agricultura. Desde los aspectos legislativos, en Nicaragua existe el Código del Trabajo que reglamenta a la Ley de Higiene y Seguridad dejó al descubierto el vacío existente en materia de formación profesional (Hedstrom, 1989). Respecto a los profesionales, agrónomos y veterinarios, particularmente los que realizan tareas de extensión a campo, es difícil percibir que profundicen en el asesoramiento de modelos preventivos a partir del conocimiento real de las consecuencias que puede tener sobre la salud de las personas las tecnologías que manejan. Ellos también son trabajadores del sector de la agricultura y en gran medida afectados por la falta de observación de las normas mínimas de prevención de riesgos en el tema específico que les ocupa: la atención sanitaria, nutricional y reproductiva en la ganadería así como el área fitopatológica de los cultivos entre otros tantos aspectos (Mamani y Merjildo, 2002).

\section{CONCLUSIONES}

- Se ha comprobado que las condiciones inadecuadas de almacenamiento, las incorrectas técnicas de manipulación y aplicación, las erróneas tácticas de eliminación de residuos físicos (envases y mezclas).

- Bajos índices de uso de equipos de protección personal se combina con la minimización por parte de los agricultores de los riesgos que ellos y sus familias, con quienes comparten trabajo y espacios.

- Estos hallazgos que llevan a la necesidad de realizar investigaciones en biota animal y vegetal, cuerpos de aguas superficiales y subterráneas.

- Un escurrimiento de plaguicidas con la adición de sales en el medio marino y cuerpos dulceacuícolas como el Rio Kama, Malopy, El Panchón puede transformar el ecosistema acuático en perjuicio de la fauna, su reproducción y fisiología de los manglares.

- Así mismo la cadena alimenticia vinculada al ecosistema manglar.

- Los plaguicidas identificados que quedan en los suelos o pasan a las masas acuáticas, pueden afectar las propiedades químicas de este y el equilibrio de sus poblaciones

- Existe la necesidad de la ejecución de capacitaciones especializadas a usuarios en medidas preventivas, la utilización de equipamiento de protección personal. 


\section{AGRADECIMIENTO}

A los agro-aplicadores, productores y técnicos extensionistas que facilitaron información crucial para la ejecución de esta investigación.

A los coordinadores de la Maestría en Medio Ambiente con especialidad en Recursos Naturales y Cuencas Hídricas:

Al MSc. René Alfonso Cassell’s Martínez de la Bluefields Indian \& Caribbean University (BICU).

Al Dr. Jordi Bartolome Firella de la Universidad Autónoma de Barcelona (UAB-España).

A la MSc. Alejandrina Herrera de Facultad Regional Multidisciplinaria, Estelí de la Universidad Nacional Autónoma de Nicaragua - Managua (FAREM - Estelí UNAN-Managua).

A la Fundación Autónoma Solidaria (FAS) de la Universitat Autònoma de Barcelona (UAB) por la ayuda concedida en las convocatorias FS XXVIII y FS XXIX.

\section{REFERENCIAS BIBLIOGRÁFICAS}

1. Albert, L. 2001. "Plaguicidas" en Introducción a la Toxicología Ambiental. Centro Panamericano de Ecología Humana y Salud. División de Salud y Ambiente. OPS y OMS., (2): 366-370; Pp. 371-376.

2. Alcaldía Municipal de Kukra Hill. 2007. Base de Datos de la Encuesta Municipal de Producción Agrícola y Pecuaria. Formato Digital PDF.

3. Bluefields Indian \& Caribbean University (BICU): Establecimiento de Proyectos Demostrativos de Buenas Prácticas agrícolas para el cultivo de la palma africana en Kukra Hill, RAAS, Nicaragua, II Fase, enero 2010. Pp. 47.

4. Bull, S., K. Fletcher, A.R. Boobis and J.M. Battershill. 2006. Evidence for genotoxicity of pesticides in pesticide applicators: a review. Mutagenesis. 21(2):93-103.

5. Bluefields Indian \& Caribbean University (BICU). 2001. Establecimiento de Proyectos Demostrativos de Buenas Prácticas agrícolas para el cultivo de la palma africana en Kukra Hill, RAAS, Nicaragua, II Fase, pp, 47.

6. Carvalho et al. 2002. Ecological risk assessment of pesticide residues in coastal lagoons of Nicaragua. $\mathrm{J}$ Environ Monit. Pp, 778-787.
7. Doog et al. 2002. Actualización de la Toxicología de los plaguicidas. Toxicología avanzada. Editorial Díaz Santos. Madrid; pp, 600.

8. Flores, A.; Zavala E. 2012. Efectos de la implementación de cobertura con frijol común (Phaseolus vulgaris) sobre el comportamiento de nutrientes en el suelo de sistemas productivos de palma africana (Elaeis guineensis) en el municipio de Kukra Hill, RAAS. Bluefields Indian \& Caribbean University (BICU). Nicaragua, pp, 117.

9. Galli, E. 2002. El empleo rural está más expuesto. En: La Nación. Buenos Aires. Argentina. 13 de abril.

10. Hedstrom, Ingemar. 1989. La situación ambiental en Centroamérica y el Caribe. San José: DEI, 320 pp, (colección ecología-teología). Antecedentes de plaguicidas en Nicaragua. Pp, 248.

11.Hilje, L; Castillo, LE; Thrupp, LA; Wesseling, I. 1987. El uso de plaguicidas en Costa Rica. San José, Costa Rica, EUNED/Heliconia.

12. Ioannis L; Robert L. 2006. Enciclopedia de salud y seguridad en el trabajo. Ministerio de Trabajo y Asuntos Sociales. España, pp, 26-28.

13. Instituto Nicaragüense de Estudios Territoriales (INETER). Dirección Recursos Hídricos.

http://www.ineter.gob.ni.

14. Kishimba M; Diaz V, Pistilli N, Guillén R, Melgarejo M, Velásquez G. 2004. Valores hematológicos en individuos expuestos accidentalmente a insecticidas organofosforados. Departamento de bioquímica. Universidad Nacional de Asunción.

15.Li, W; Eddleston M, Eyer P, Worek F, Mohammed F, Senarathna L, Meyer L, et al. 2001. Differences between Organophosphorus Insecticides in Human Self-Poisoning. Lancet, pp, 68-83.

16. Mamani, F.; Merjildo, D. 2002. Universidad peruana Unión. Diagnóstico situacional: medidas preventivas aplicadas por los agricultores y actividades de prevención primaria que realizan los profesionales de salud frente al uso de plaguicidas en el valle de Chillón durante el año 2002. Boletín RAAA, pp, 202.

17. Miller, G. (2004). Sustaining the Earth, 6th edition. Thompson Learning, Inc. Pacific Grove, California. Chapter 9, pp, 119-180.

18. Malik L. Schaefer M. 2010. Guías para el Fortalecimiento de los Servicios de Salud Ambiental hacia una Mejor Acción Gubernamental en los Determinantes Ambientales de la Salud. Ginebra. Pp, 280.

19. Mamani, F.; Merjildo, D. 2002. Universidad peruana Unión. Diagnóstico situacional: medidas preventivas aplicadas por los agricultores y actividades de prevención primaria que realizan los profesionales de 
salud frente al uso de plaguicidas en el valle de Chillón durante el año 2002. Boletín RAAA. Pp. 202.

20. Organización de las Naciones Unidas para la Agricultura y Alimentación. 2007. Código Internacional de conducta para la Distribución y Utilización de Plaguicidas. Roma, pp, 237.

21.Programa de las Naciones Unidas para el Medio Ambiente (PNUMA). 2011. Informe final proyecto regional Reducción del escurrimiento de plaguicidas al mar Caribe. Kingston, Jamaica, pp, 45

22. Romero T. 2010 C. Determinación de inmunoglobulinas en obreros manipuladores de plaguicidas. Rev Cubana de Salud y trabajo, pp, 32.

23.Sarkar J. MacArthur I, Bonnefoy X. 2008. Environmental health services in Europe: an overview of practice in the 1990's. WHO/EURO. Copenhagen.

24. Turnbull et al. 1985. Consejo de Salud Ocupacional. Proyecto de la OIT con sede en Costa Rica: la seguridad y salud de los trabajadores agrarios de América Central. Bol Info (San José).

25. Vílchez, R. 2009. Situación actual del combate de nematodos en plantaciones de banano en Costa Rica, pp, 75-76. In: Informe anual Dirección de Investigaciones, CORBANA.

26. Wesseling, C. 1994. Uso de plaguicidas en América Central y su impacto en la salud de los trabajadores. Programa de Plaguicidas PNUMA. Escuela de Ciencias Ambientales. Universidad de Costa Rica.

27. WorkingLife. 1999. Gramoxone: una peligrosa realidad en Costa Rica. LA NACIÓN, San José, Costa Rica, pp, 15.

28. Wright D., Welbourn P. 2002. Environmental Toxicology and Chemistry Great Lakes hiomonitoring: Ryckman, DP, D. V C. Weseloh, and CA Bishop, pp, 981.

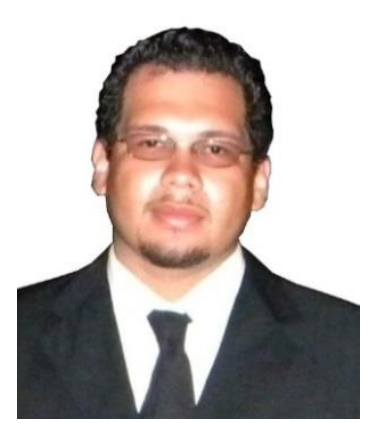

Asdrúbal Flores Pacheco:
Máster en Medio Ambiente
por la Universidad Autónoma
de Barcelona (UAB), España
(2013). Ingenieror en
Agroecología Tropical por la
Universidad Nacional
Autónomo de Nicaragua -
León (UNAN-León) (2010).
Docente horario de la Facultad de Recursos Naturales y Medio Ambiente (FARENA) desde 2011. Para contactos remirase a la oficina de FARENA, campus BICU, Avenida Universitaria, Barrio San Pedro, Bluefields, correo asdrubalflorespacheco@gmail.com.
Álvaro Mairena Valdivia: Máster en Pedagogía Universitaria por la Universidad Nacional Autónomo de Nicaragua - Managua (UNAN-Managua). Licenciado en Biología por la Universidad Nacional Autónomo de Nicaragua - León (UNAN-León). Docente con más de 29 años de experiencia, Secretario Académico de la Facultad de Recursos Naturales y Medio Ambiente (FARENA) de la Bluefields Indian \& Caribbean University. Para contactos remirase a la oficina de FARENA, campus BICU, Avenida Universitaria, Barrio San Pedro, Bluefields, correo amava7@ gmail.com.

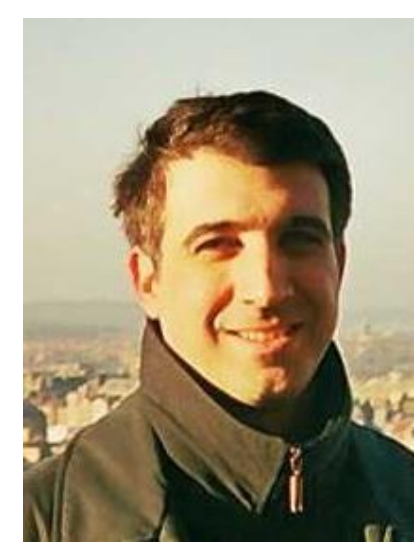

Josep Espluga: Doctor en Sociología por la Universidad Autónoma de Barcelona y Master en Prevención de Riesgos Laborales por la Universidad Politécnica de Catalunya. Profesor del Departamento de Sociología de la UAB. Anteriormente fue investigador del Centro Nacional de Condiciones de Trabajo (Instituto Nacional de Seguridad e Higiene en el Trabajo), Fundación para la Mejora de la Asistencia Sanitaria. Sus líneas de investigación se centran en una aproximación sociológica a las relaciones entre salud y trabajo, territorio y medio ambiente y, más específicamente, sobre la gobernanza de los riesgos tecnológicos. Para contactos remirase al Departamento de Sociología, Universidad Autónoma de Barcelona, España Campus de la UAB; 08193 Bellaterra, correo joseplluis.espluga@uab.cat. 